\title{
Abordaje multidisciplinar en el tratamiento del cáncer de recto: eficacia del tratamiento neoadyuvante
}

\author{
P. Priego, A. Sanjuanbenito, V. Morales, E. Lobo, E. Martínez Molina, G. Rodríguez Velasco y V. Fresneda \\ Departamento de Cirugía General y Digestivo. Hospital Universitario Ramón y Cajal. Madrid
}

\section{RESUMEN}

Objetivo: evaluar el papel de la terapia neoadyuvante en el tratamiento del cáncer de recto en nuestro hospital, tras la implantación de un grupo multidisciplinar de trabajo (MDT).

Material y métodos: desde enero de 2000 hasta diciembre de 2005 se ha evaluado en sesión clínica por el grupo MDT un total de 90 pacientes diagnosticados de adenocarcinoma de recto $e$ intervenidos quirúrgicamente tras recibir tratamiento neoadyuvante con radioquimioterapia (QMRT). La edad media fue de 65,04 años (21-83 años), siendo un $67 \%$ varones y un $33 \%$ mujeres. Los procedimientos quirúrgicos realizados fueron fundamentalmente resección anterior baja en un $50 \%$ y amputación abdominoperineal en un $42,2 \%$.

Resultados: la tasa de complicaciones asociada al uso del tratamiento neoadyuvante fue del $54,44 \%$, siendo más frecuentes las gastrointestinales. Sin embargo, esta toxicidad fue bien tolerada en la mayor parte de los casos, siendo grave y acarreando la suspensión del tratamiento quimioterápico en 2 pacientes $(2,2 \%)$. El análisis anatomopatológico de las piezas resecadas demostró una respuesta completa en 10 casos $(11,1 \%)$ y una respuesta parcial (disminución del parámetro T de la clasificación TNM) en 32 casos $(35,6 \%)$, con lo que la respuesta global del tratamiento neoadyuvante fue del $46,6 \%$. Entre las complicaciones postoperatorias se produjeron un $8,3 \%$ de dehiscencias anastomóticas, un $34,2 \%$ de complicaciones de la herida perineal y un $12,2 \%$ de complicaciones urinarias. La mortalidad quirúrgica fue del $0 \%$. La tasa de recidiva pélvica fue del $4,4 \%$ y la sistémica del $22,2 \%$. La supervivencia actuarial global y libre de enfermedad a los 5 años fue del 80 y $64 \%$ respectivamente.

Conclusión: el tratamiento QMRT neoadyuvante proporciona una cifras bajas de recidiva pélvica junto con buenas tasas de supervivencia, no añadiendo una morbimortalidad importante al acto quirúrgico. La evaluación sistemática por un grupo MDT en el contexto de un protocolo clínico parece ofrecer al paciente mejores oportunidades de curación.

Palabras clave: Cáncer de recto. Neoadyuvancia. Radioterapia. Quimioterapia. Supervivencia. Recidiva. Respuesta patológica. Abordaje multidisciplinar.

Recibido: 31-01-08.

Aceptado: 21-04-08.

Correspondencia: Pablo Priego Jiménez. C/ Fermín Caballero, 26, $1^{\circ}$ A. 16004 Cuenca.e-mail: papriego@hotmail.com

\begin{abstract}
Objective: the aim of this study was to assess the impact of neoadjuvant treatment on rectal cancer following involvement of a multidisciplinary team (MDT).

Materials and methods: between January 2000 and December 2005, 90 patients with rectal adenocarcinoma were evaluated by a MDT and operated on after receiving neoadjuvant treatment with radiochemotherapy (RTCT) $-67 \%$ were men and $33 \%$ were women, with a mean age of 65.04 years $(21-83$ years). Surgery was low anterior resection in $50 \%$ and abdominoperineal amputation in $42.2 \%$.

Results: the rate of complications associated with neoadjuvant treatment was $54.4 \%$, with gastrointestinal complications being most frequent. However, this toxicity was tolerated by most patients. It was severe in two cases $(2.2 \%)$, leading to chemotherapy discontinuation. A histological analysis of specimens showed a complete pathologic response in 10 cases $(11.1 \%)$ and a partial response (downstaging of $\mathrm{T}$ ) in 32 cases $(35.6 \%)$, hence overall response to neoadjuvant treatment was $46.6 \%$. Postoperative complications included anastomotic leakage in $8.3 \%$, perineal wound complications in $34.2 \%$, and urinary disease in $12.2 \%$. The surgical mortality rate was $0 \%$. Local recurrence occurred in $4.4 \%$, and distant metastases were found in $22.2 \%$. Both overall and disease-free survivals were 80 and $64 \%$, respectively.

Conclusions: neoadjuvant treatment results in low local recurrence rates and optimal survival rates, with no increase in morbidity or mortality. A systematic evaluation by a MDT in the context of a clinical protocol offers better cure rates.
\end{abstract}

Key words: Rectal cancer. Neoadjuvant therapy. Radiotherapy. Chemotherapy. Survival. Recurrence. Pathological response. Multimodality approach.

Priego P, Sanjuanbenito A, Morales V, Lobo E, Martínez Molina $E$, Rodríguez Velasco G, Fresneda V. Abordaje multidisciplinar en el tratamiento del cáncer de recto: eficacia del tratamiento neoadyuvante. Rev Esp Enferm Dig 2008; 100: 393-399. 


\section{INTRODUCCIÓN}

A pesar de que la cirugía continúa siendo hoy en día el tratamiento de elección del cáncer de recto (1), el abordaje de esta patología debe ser multidisciplinar, jugando un papel importante, además del cirujano, los oncólogos, patólogos, radiólogos y oncólogos radioterapeutas $(2,3)$.

El objetivo del tratamiento consiste en definitiva en disminuir la tasa de recidivas (que supone la causa más frecuente de mortalidad de estos pacientes) y aumentar la supervivencia (4).

En las últimas décadas, han sido muchos los trabajos publicados $(5,6)$ que han demostrado la importancia del tratamiento neoadyuvante con radioquimioterapia (QMRT) a la hora de reducir no sólo la tasa de recurrencias locales sino en algunos casos también producir un aumento de la supervivencia (7-9).

De forma paralela, la mejora en las técnicas radioterápicas y los nuevos agentes quimioterápicos han conseguido disminuir la toxicidad del tratamiento neoadyuvante y han aumentado las tasas de respuesta patológica, por lo que hoy en día está aceptado como tratamiento de elección junto con la cirugía en los tumores de recto en estadios II-III.

El objetivo del trabajo es efectuar una revisión de la eficacia de la QMRT preoperatoria en el tratamiento del cáncer de recto, haciendo especial hincapié en la respuesta del tumor al tratamiento neoadyuvante, su impacto sobre la recidiva locorregional, supervivencia y la posibilidad de efectuar una cirugía radical con preservación esfinteriana, desde la implantación en nuestro hospital de un grupo multidisciplinar de trabajo en el cáncer colorrectal (MDT).

\section{MATERIAL Y MÉTODOS}

Desde enero de 2000 hasta diciembre de 2005 se evaluó de forma prospectiva un total de 104 pacientes diagnosticados de adenocarcinoma de recto e intervenidos quirúrgicamente tras recibir tratamiento neoadyuvante con QMRT. Excluimos a 14 pacientes debido a la presencia de enfermedad metastásica o existencia de tumor irresecable en el momento de diagnóstico preoperatorio, de modo que el estudio incluye a un total de 90 pacientes.

Todos los pacientes fueron discutidos en sesión por el MDT, decidiendo dicho comité las actuaciones diagnósticas y terapéuticas a seguir en cada uno de los casos. Este grupo está compuesto por cirujanos, oncólogos, oncólogos radioterapeutas, gastroenterólogos, radiólogos y patólogos.

\section{Evaluación preoperatoria del paciente con cáncer de recto}

\section{Preoperatorio básico}

Además de una historia clínica y exploración física completa (incluido tacto rectal), en todos los pacientes se efectuó una radiografía de tórax (para descartar metástasis pulmonares), un electrocardiograma y una analítica de sangre con determinación de los niveles de antígeno carcinoembrionario (CEA).

\section{Colonoscopia}

La realización de una colonoscopia completa es esencial en el diagnóstico preoperatorio de estos pacientes, ya que no sólo consigue visualizar el tumor, sino que permite la toma de biopsias para efectuar el estudio histológico del mismo. En aquellos casos en los que la colonoscopia completa no pudo realizarse, bien por imposibilidad para introducir el endoscopio en caso de tumores obstructivos o por mala tolerancia del paciente, se efectuó un enema opaco para descartar la existencia de una lesión sincrónica colónica (5\% de los casos) (10).

\section{Ecografía endorrectal}

Todos los pacientes fueron estudiados mediante ecografía endorrectal realizada por los investigadores y estadiados preoperatoriamente (uTuN) según la profundidad de la afectación de la pared rectal y la presencia de adenopatías sospechosas de metástasis. El equipo utilizado fue un transductor de $360^{\circ}$ y $10 \mathrm{MHz}$ de frecuencia. El cáncer de recto aparece como una imagen hipoecoica que se origina en la mucosa e infiltra las diferentes capas de la pared de dentro a fuera. Según la clasificación TNM, las lesiones uT1 indican que el tumor está confinado a la mucosa-submucosa, las uT2 implican la penetración en la muscular propria, las uT3 denotan una invasión de la grasa perirrectal y las uT4 una extensión a órganos vecinos. Nuestra experiencia previa arroja una sensibilidad del $80 \%$ para la $\mathrm{T}$ y del $60 \%$ para la $\mathrm{N}$.

\section{Tomografía computerizada abdominopélvica (TAC)}

De forma sistemática se efectúa una tomografía abdominal a todos los pacientes, no sólo para excluir la existencia de metástasis hepáticas, sino también para determinar la relación del tumor con las estructuras pélvicas adyacentes.

\section{Tratamiento neoadyuvante}

En base al protocolo de actuación en el tratamiento del cáncer de recto existente en nuestro hospital, recibieron tratamiento neoadyuvante con QMRT los pacientes con estadios tumorales T3-4 o N1, o bien aquellos con un tumor circunferencial, voluminoso o fijo en el tacto rectal.

La dosis total de radioterapia (RT) utilizada fue de $45 \mathrm{~Gy}$, repartidos en 180 cGy al día durante 5 semanas. 
De forma paralela se administraron dos ciclos de quimioterapia (QM) con 5-fluoracilo y ácido folínico.

\section{Tratamiento quirúrgico}

La cirugía se efectuará aproximadamente entre 4-6 semanas después de completar el tratamiento con RT. El tratamiento quirúrgico consiste en la realización de una resección anterior o una amputación abdominoperineal, efectuando la disección del recto siguiendo el plano existente entre la fascia endopélvica y la fascia visceral del recto.

Para el análisis estadístico de los resultados se utilizó el programa SPSS (versión 14.0), analizando las variables cualitativas mediante el test de la Chi cuadrado y el test de Kaplan Meyer para el estudio de la supervivencia. Se considera que el resultado es estadísticamente significativo cuando la $\mathrm{p}<0,05$.

\section{RESULTADOS}

De los 90 pacientes que participaron en el estudio, 60 eran varones y 30 mujeres. La edad media fue de 65,04 años, con un rango de 21-83 años. El tumor se localizó en el tercio superior $(11-15 \mathrm{~cm})$ en 12 casos, en el tercio medio $(6-10 \mathrm{~cm})$ en 44 y en el tercio inferior $(0-5 \mathrm{~cm})$ en 34 .

Las características anatomopatológicas del tumor se describen en la tabla I.

Tabla I. Características anatomopatológicas del tumor

\begin{tabular}{lc}
\hline & Número de pacientes \\
\hline Clasificación Dukes & 18 \\
A & 44 \\
B & 19 \\
C (N positivos) & \\
Grado de diferenciación & 73 \\
Bien & 5 \\
Moderado & 1 \\
Pobre & $496(6,62$ ganglios por paciente) \\
Número de ganglios en pieza quirúrgica & 49 \\
Número de ganglios positivos en pieza quirúrgica & 11 \\
Componente mucinoso & 3 \\
Sí > 50\% & 66 \\
Sí < 50\% & $21(23,3 \%)$ \\
No & \\
Infiltración linfática, venosa y perineural & \\
\hline
\end{tabular}

Se produjo toxicidad asociada al tratamiento neoadyuvante en 49 casos $(54,4 \%)$. Los efectos secundarios más frecuentes son a nivel gastrointestinal 43 casos, cutáneo 8 casos, neurológico 4 casos, vesical 4 casos y hematológico 3 casos. Sin embargo, esta toxicidad fue bien tolerada en la mayor parte de los casos, siendo grave y acarreando la suspensión del tratamiento quimioterápico en 2 pacientes $(2,2 \%)$. Un paciente desarrolló una crisis comicial secundaria a trombosis cerebral y otro paciente un tromboembolismo pulmonar con trombosis del catéter.
Se practicó resección anterior baja $(\mathrm{RAB})$ en 45 pacientes $(50 \%)$ y amputación abdominoperineal (AAP) en 38 pacientes $(42,2 \%)$. Otras intervenciones fueron: colostomía de descarga en 2 pacientes (después de esta primera cirugía y tras recibir tratamiento con QMRT, al visualizar regresión de la enfermedad, se decidió reintervenir a los pacientes, extirpando completamente el tumor, en un caso mediante una exanteración pélvica y en otro caso mediante una AAP), exanteración pélvica posterior (1), intervención de Hartmann (1), hemicolectomía izquierda (1), proctectomía (1) y proctocolectomía (1). Todas las intervenciones fueron efectuadas a través de una laparotomía, no realizándose ninguna por laparoscopia.

La duración media de la cirugía fue de 193,68 minutos con un rango de 80-340 minutos y la estancia media postoperatoria de 19,51 días con un rango de 4-127 días.

Entre los 48 pacientes en los cuales se efectuó una resección más anastomosis cólica, se realizó ileostomía de protección en 31 casos $(64,6 \%)$, cerrándose el estoma en una segunda intervención en todos los casos.

La tasa de complicaciones postoperatorias fue del 52,2\% (47 en 90 pacientes), siendo las más importantes la dehiscencia de la anastomosis en un 8,3\% (4 de 48 pacientes), las complicaciones de la herida perineal en un $34,2 \%$ (13 de 38 pacientes) y las complicaciones urinarias en un 12,2\% (11 de 90: ITU, RAO, vejiga arrefléxica, uropatía obstructiva, incontinencia, disfunción eréctil, fístula uretra,...).

La tasa de mortalidad postoperatoria fue del $0 \%$, reinterviniéndose durante el ingreso a 6 pacientes $(6,6 \%)$.

En 55 pacientes $(61,1 \%)$ se administró tratamiento adyuvante posterior a la cirugía, siendo quimioterapia en 47 casos, quimioterapia y radioterapia en 5 casos y radioterapia aislada en 1 caso.

El análisis anatomopatológico de las piezas resecadas demostró una respuesta completa (ausencia del tumor en la pieza quirúrgica ${ }_{\mathrm{P}}$ TONOM0) en 10 casos $(11,1 \%)$ y una respuesta parcial (disminución del parámetro $\mathrm{T}$ de la clasificación TNM) en 32 casos $(35,6 \%)$, con lo que la respuesta global del tratamiento neoadyuvante fue del $46,6 \%$ (Tabla II).

Tabla II. Análisis anatomopatológico de las piezas resecadas

\begin{tabular}{lcccccc}
\hline & T0 & T1 & T2 & T3 & T4 & Total \\
\hline Estadiaje preoperatorio & 0 & 1 & 6 & 80 & 3 & 90 \\
Estadiaje postoperatorio & 10 & 6 & 28 & 43 & 3 & 90 \\
\hline
\end{tabular}

En ningún paciente se observó afectación del margen circunferencial, siendo este superior a $1 \mathrm{~mm}$ en todos los casos.

Al analizar si existen diferencias en cuanto a la respuesta al tratamiento neoadyuvante, no se observan diferencias estadísticamente significativas en ninguno de los parámetros analizados, si bien se observa una mayor su- 
pervivencia en términos absolutos en aquellos pacientes en los cuales se había producido una respuesta patológica completa en comparación con los que habían tenido una respuesta parcial y o no habían respondido al tratamiento $(\mathrm{p}=0,82)$ (Fig. 1).

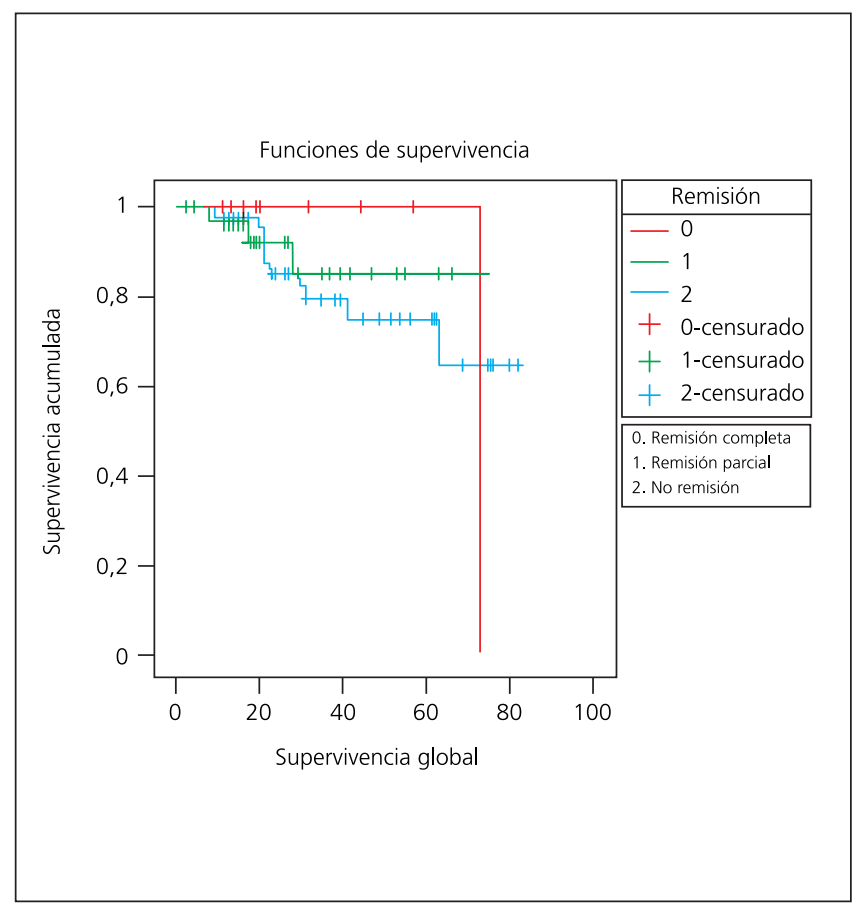

Fig. 1. Relación supervivencia-respuesta al tratamiento.

Durante el periodo de seguimiento (33,4 meses de media, rango 12-82 meses), se produjo recidiva locorregional en 4 pacientes, lo que supone una tasa del $4,4 \%$, y recidiva sistémica en 20 pacientes $(22,2 \%)$. De los 4 pacientes con recidiva, sólo se pudo rescatar quirúrgicamente a uno de ellos (25\%), que había desarrollado una recidiva perineal tras una AAP.

Únicamente hay dos parámetros que presentan diferencias estadísticamente significativas en cuanto a la supervivencia: la presencia de infiltración venosa, linfática y perineural se asocia con mayor frecuencia de recidiva frente a no tener infiltración $(43 v s .22 \%, \mathrm{p}=0,031)$ y los pacientes con ganglios positivos en la pieza tienen mayor porcentaje de recidivas frente a los que tienen ganglios negativos tras el tratamiento neoadyuvante $(\mathrm{N}+\mathrm{t}$ 36,8\% vs. $\mathrm{N}-: 0 \%, \mathrm{p}=0,022)$.

La supervivencia global y libre de enfermedad a los 5 años fue del 80 y $64 \%$ respectivamente, siendo la supervivencia global media de 33,4 meses (rango 4-82 meses) y la supervivencia libre enfermedad media de 27,8 meses (rango 1-80 meses) (Figs. 2 y 3).

$\mathrm{Al}$ analizar si existen diferencias en supervivencia en relación a los diferentes parámetros, observamos que el único factor que presenta significación estadística es la recidi-

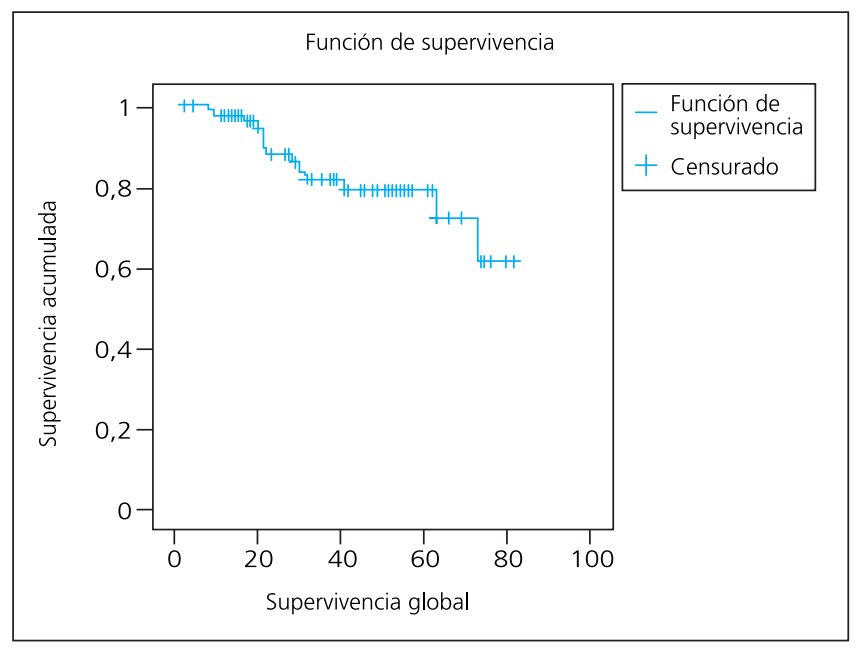

Fig. 2. Supervivencia global.

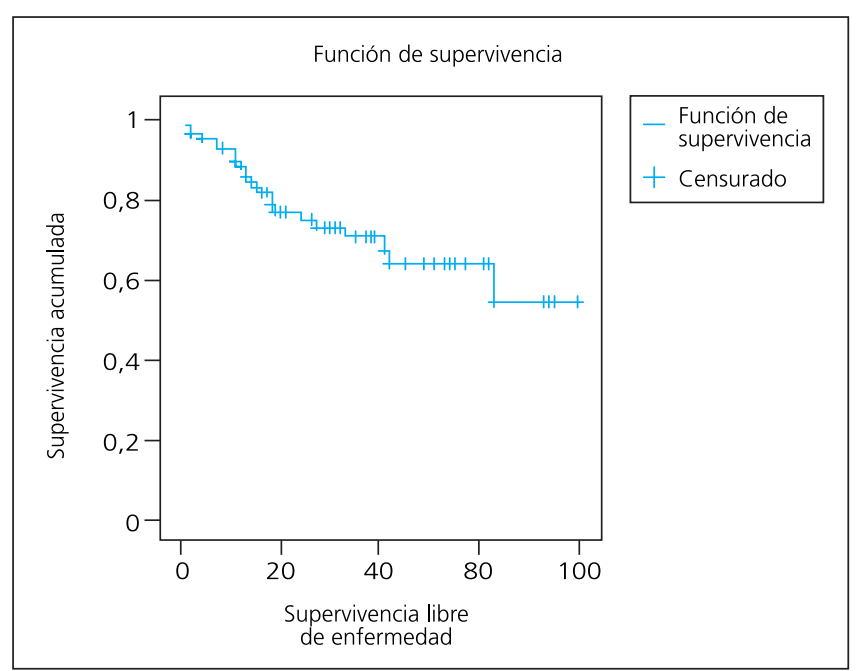

Fig. 3. Supervivencia libre de enfermedad.

va, siendo la supervivencia mayor en los pacientes en los cuales el tumor no ha recidivado ( $\mathrm{p}=0,0018)$ (Fig. 4).

A pesar de la administración de tratamiento neoadyuvante, en los pacientes con tumores localizados en el recto inferior, se efectuó una AAP en un 73,5\% de los casos (Tabla III).

\section{DISCUSIÓN}

A pesar de que la cirugía continúa siendo hoy en día el tratamiento de elección del cáncer de recto (1), el abordaje de esta patología debe ser multidisciplinar, jugando un papel importante además del cirujano, los oncólogos, patólogos, gastroenterólogos, radiólogos y oncólogos radioterapeutas $(2,3)$. 


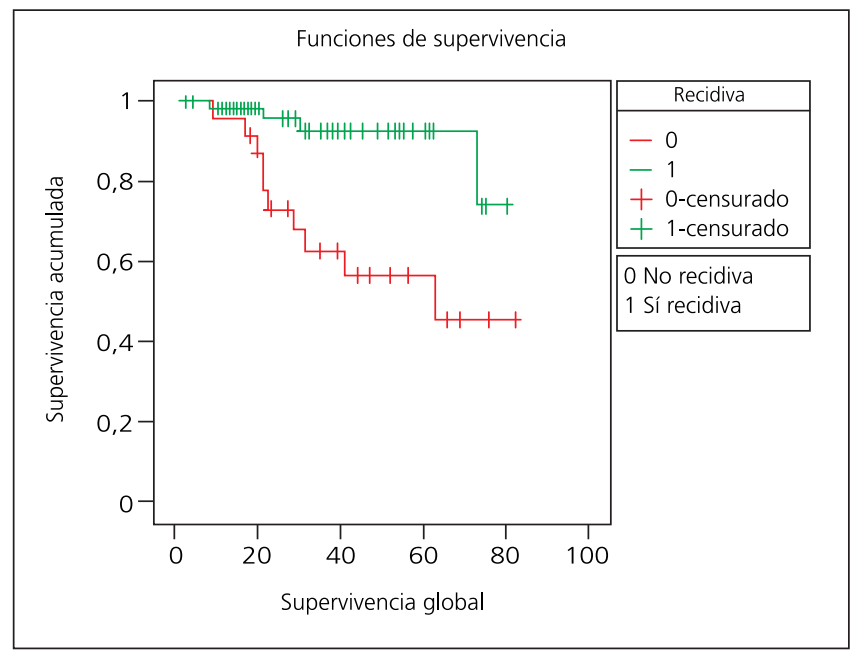

Fig. 4. Relación supervivencia-recidiva.

Tabla III. Relación de tratamiento neoadyuvante con preservación de esfínteres

\begin{tabular}{lccc}
\hline & RAB & AAP & Otras \\
\hline Recto sup. $(11-15 \mathrm{~cm})$ & 10 & 1 & 1 \\
Recto med. $(6-10 \mathrm{~cm})$ & 28 & 12 & 4 \\
Recto inf. $(0-5 \mathrm{~cm})$ & $7(20,58 \%)$ & $25(73,53 \%)$ & 2 \\
\hline
\end{tabular}

Aunque no existe ninguna evidencia de un menor coste-beneficio ni de una mayor supervivencia de los pacientes en los cuales el manejo diagnóstico y terapéutico se efectúa a través de un equipo multidisciplinar, resulta un hecho evidente que la calidad del tratamiento efectuado debe ser mayor cuando un grupo de expertos en el cáncer colorrectal se reúnen y discuten todos los detalles relacionados con el tipo de tratamiento que va a recibir cada paciente (3). Así pues, todos nuestros pacientes fueron discutidos individualmente por el comité, decidiendo el tratamiento más apropiado en cada caso en base al protocolo de actuación en el tratamiento del cáncer de recto existente en nuestro hospital.

El procedimiento de estadificación local del cáncer de recto está sujeto a bastantes controversias. El problema radica en la posibilidad de infraestadiar a los pacientes, lo que conduciría a que el paciente recibiera un tratamiento insuficiente en la curación de su patología. En definitiva, y para evitar esto, tendemos a sobreestadiar a los pacientes y en consecuencia a sobretratar a los mismos. El objetivo del estadiaje consistiría en identificar a los pacientes que podrían beneficiarse del tratamiento combinado, descartando a aquellos en los que el beneficio no supera los efectos nocivos (1).

Nuestro grupo es partidario del uso de la ecografía endorrectal (EE) como herramienta en el estadiaje preoperatorio, pues aunque es menos útil en el diagnóstico de las metástasis ganglionares, con una sensibilidad del 60$80 \%$, resulta extremadamente útil en el diagnóstico de la penetración del tumor en la pared rectal (con una sensibilidad entre el 80-90\%) $(11,12)$.

Aunque la EE ha demostrado ser superior a la TAC en el diagnóstico preoperatorio de los tumores de recto, por su mayor fiabilidad en el estadiaje de la $\mathrm{T}$ y la N, la TAC ha demostrado su utilidad conforme aumenta el estadio del tumor (localmente avanzados) y permite valorar el hígado, por lo que consideramos que es una buena prueba complementaria en el estadiaje preoperatorio de los pacientes.

En nuestro centro no se dispone de resonancia magnética $(\mathrm{RMN})$ para el estadiaje preoperatorio del cáncer de recto. Diferentes autores han señalado que esta ofrece como ventajas visualizar con nitidez la relación del tumor primario y los ganglios linfáticos del mesorrecto con la fascia rectal así como predecir con certeza la relación del tumor con el margen circunferencial (MRC). Diversos estudios de Bisset y cols. (13) y Brown y cols. (14) demuestran una concordancia entre la RMN y la histología en el MRC del 92-95\%, así como una sensibilidad y especificidad de la RMN para describir un MRC sano del 80 y $92 \%$ respectivamente $(11,15)$. Los que abogan por la realización de la RMN aducen que la mejor identificación del MRC va a poder determinar de forma de una forma más precisa cuáles van a ser los pacientes que más van a beneficiarse de un tratamiento neoadyuvante de forma previa a la cirugía, obteniendo mejores resultados en cuanto a costes y eficacia clínica en comparación con el tacto rectal y la EE (16).

Kim y cols. (11) comparan el papel de la EE, la TAC y la RMN, y observan que la fiabilidad de la EE y la RMN en la determinación de la $\mathrm{T}$ son similares (en torno al $81 \%$ ) y superiores a la TAC (65\%), mientras que la identificación de la $\mathrm{N}$ fue igualmente pobre en las tres modalidades $(64,57$ y $63 \%$ para la EE, TAC y RMN).

Si bien la EE puede ser mejor que la TAC y la RMN en la evaluación de la $\mathrm{T}$, las otras técnicas son superiores a la EE en estadios avanzados de la enfermedad. Por tanto, nosotros consideramos que tanto la EE, como la TAC y la RMN son técnicas complementarias y no excluyentes.

La controversia existente en torno al uso de RT pre- o postoperatoria parece haberse solucionado a favor de la primera, pues no sólo se han demostrado menores tasas de recurrencia y complicaciones, sino también una mayor tasa de supervivencia y de respuestas al tratamiento (1719), si bien todavía existe controversia acerca del uso del ciclo corto o largo de RT o a la utilización combinada con RT y QM $(8,20)$.

En nuestra experiencia, la administración de un ciclo largo de RT combinada con QM no ha presentado una alta tasa de complicaciones ni ha añadido morbimortalidad al acto quirúrgico. Como hemos comentado previamente, la mayor parte de las mismas han sido gastrointestinales (diarrea, vómitos,...) y han cedido con tratamiento sintomático. Si comparamos las mismas con otros estudios $(7,8,21,22)$, observamos que en nuestra serie son ligeramente superio- 
res, pero podría estar en relación a que en nuestro protocolo se utiliza el ciclo largo de radioterapia con 45 Gy durante 5 semanas y en los otros se utiliza el ciclo corto con 25 Gy durante una semana. En estos últimos, como la mayor parte de los pacientes se opera una semana después de la RT, no da tiempo a que aparezcan los síntomas. Además, en nuestra serie, sólo en un 2,2\% hubo que suspender el tratamiento debido a complicaciones mayores, lo cual, dado el gran beneficio global que ha supuesto en nuestra experiencia el uso del tratamiento neoadyuvante, no ha reducido la utilización del mismo.

Recientemente estudios suecos y holandeses $(23,24)$ han publicado los efectos a largo plazo del tratamiento neoadyuvante con RT (ciclo corto), señalando que los pacientes radiados tienen un mayor número de disfunciones intestinales (incontinencia, pérdidas de sangre y moco, uso de pañales), pero que su calidad de vida es buena y no es significativamente diferente a la de los pacientes no radiados. Su conclusión es que, aunque el tratamiento neoadyuvante incrementa el control local de la enfermedad, las posibles complicaciones a largo plazo hacen que en el futuro se deban establecer estrategias para seleccionar a los pacientes con alto riesgo de recidiva. El poco tiempo de seguimiento de nuestros pacientes no nos permite hablar de complicaciones a largo plazo.

En cuanto al tema de la recurrencia tras la cirugía curativa, tras un periodo de seguimiento de 33,4 meses, hemos conseguido unas cifras bajas de recidiva pélvica $(4,4 \%)$. Dado que aproximadamente el $80 \%$ de las recurrencias ocurre en los primeros 24 meses de seguimiento, creemos que nuestros datos se aproximan bastante a la realidad. Estos resultados son bastante buenos si se comparan con las cifras existentes en la literatura, que oscilan entre el 3-30\% $(8,20,25)$. Probablemente en este punto han podido influir no sólo los efectos de la RT sino también la mayor especialización del equipo quirúrgico. El análisis de los diferentes factores únicamente ha encontrado aumento de la tasa de recidiva en los pacientes con infiltración venosa, linfática y perineural y en los pacientes con ganglios positivos en la pieza tumoral (C de Dukes).

En cuanto al tema de la respuesta al tratamiento neoadyuvante, es un hecho conocido que tras la administración de RT (ciclo largo) se va a producir una disminución/desaparición del tumor, con cifras de respuesta patológica completa en torno al 5-30\%. En nuestra casuística, la respuesta global al tratamiento neoadyuvante fue en torno al $47 \%$, con una respuesta patológica completa del $11 \%$, que está dentro de las cifras encontradas en la literatura (2,26-33), si bien hemos observado un aumento de esta cifra en comparación con los resultados obtenidos en nuestro hospital entre los años 1995-2002 (4,7\%), pudiendo ser debido no sólo a la mayor experiencia, sino también a la mejora en el equipo de radioterapia e instalaciones desarrollada en los últimos años, así como a la utilización de nuevos agentes quimioterápicos. Si comparamos nuestros resultados con otros autores (26-33), observamos que algunos de ellos presentan cifras de res- puesta tras el tratamiento neoadyuvante mayores que las nuestras. Tenemos que comentar que eso se debe a la diferente forma de evaluar la respuesta al tratamiento neoadyuvante. En nuestra serie hemos considerado exclusivamente como respuesta al tratamiento la disminución del parámetro $\mathrm{T}$ con el tratamiento neoadyuvante. De esta forma, es posible que, aunque un tumor haya disminuido de volumen tras el tratamiento, la simple presencia de un nido de células tumorales en la grasa perirrectal de la pieza quirúrgica hace que consideremos que dicho tumor no ha respondido al tratamiento.

Las cifras obtenidas en cuanto a supervivencia global y libre de enfermedad a los 5 años, en torno al 80 y $64 \%$ respectivamente, son bastante interesantes en comparación con otros grupos $(5,8,9,20)$. El único factor que ha parecido influir en la supervivencia, como era de esperar, es la existencia de recidivas, pues no en vano es la causa más frecuente de mortalidad en estos pacientes. No hemos encontrado diferencias en supervivencia con respecto al sexo, localización del tumor, estadio de Dukes preoperatorio y características anatomopatológicas, tipo de operación efectuada y remisión tras el tratamiento neoadyuvante,...

Por último, la posibilidad de preservación esfinteriana en el tratamiento quirúrgico del cáncer de recto con la administración del tratamiento neoadyuvante también es un tema controvertido, con resultados dispares en la literatura. El índice de preservación esfinteriana oscila entre un 23$89 \%$ con una media del 63\% (33-35). A pesar de que el diseño y el objetivo del trabajo no está dirigido a solucionar dicho problema (ya que la indicación de AAP o cirugía conservadora de esfínteres se realiza de forma previa a la administración de neoadyuvancia y no se modifica aunque haya habido respuesta), en base a nuestros resultados, creemos que el tratamiento neoadyuvante no va a conseguir aumentar la tasa de preservación esfinteriana, ya que, en nuestra serie, en un 73,5\% de los tumores de recto inferior se efectuó una amputación abdominoperineal. En nuestra experiencia, la tasa de preservación esfinteriana es por tanto del $58 \%$. Sin embargo, creemos que la decisión de efectuar una cirugía conservadora de esfínteres no debe estar marcada por la destreza del equipo quirúrgico ni por los efectos del tratamiento neoadyuvante, sino por las características del paciente. Muchos pacientes presentan previamente a la cirugía problemas de continencia y, al efectuarles una resección ultrabaja con preservación esfinteriana, probablemente curemos su enfermedad, pero les creamos importantes alteraciones de su calidad de vida. De ahí que pensamos que no se debe efectuar una conservación a ultranza de los esfínteres, sino que se deben tener otros factores a la hora de plantear la cirugía.

Como crítica al trabajo se podría decir que, para obtener unos resultados más concluyentes y con mayor significación estadística, se tendría que incluir un mayor número de pacientes y efectuar un mayor tiempo de seguimiento. Sin embargo, a pesar de estos dos factores en contra, creemos que nuestros resultados están acordes con los descritos en la literatura. 
En el futuro se deben seguir mejorando las pruebas de estadificación preoperatorias para evitar la sobreestadificación y en consecuencia el sobretratamiento y avanzar en el estudio molecular de los tumores con el objetivo de seleccionar a aquellos pacientes en los que el beneficio del tratamiento no supere los efectos nocivos del mismo (1). También sería interesante conocer los resultados a largo plazo del grupo brasileño (2), pues podría revolucionar el abordaje terapéutico del tratamiento del cáncer de recto.

Para finalizar, y como conclusión, en nuestra experiencia el tratamiento QMRT neoadyuvante proporciona unas cifras bajas de recidiva pélvica junto con buenas tasas de supervivencia, no añadiendo una morbimortalidad importante al acto quirúrgico. La tasa de respuesta patológica completa tras la neoadyuvancia está dentro de las cifras descritas en la literatura y en nuestra experiencia en los tumores del recto inferior la neoadyuvancia no mejora la tasa preservadora de esfínteres.

\section{BIBLIOGRAFÍA}

1. Espín E, Armengol M. Radioterapia en el cáncer de recto. Estado actual. Cir Esp 2004; 76 (6): 347-52.

2. Habr-Grama A, Oliva R, Nadalin W, et al. Operative versus nonoperative treatment for stage 0 distal rectal cancer following chemoradiation therapy. Ann Surg 2004; 240: 711-8.

3. Cervantes A, Chirivella I, Rodríguez-Braun E, et al. A multimodality approach to localizad rectal cancer. Ann Oncology 2006; 17 (Supl. 10): 129-34.

4. Ramírez-Rodríguez JM, Aguilella-Diago V. Recidiva local en el cáncer de colon y recto. Cir Esp 2005; 78 (6): 344-50.

5. Colorectal Cancer Collaborative Group. Adjuvant radiotherapy for rectal cancer: A systematic overview of 8507 patients from 22 randomised trials. Lancet 2001; 358: 1291-304.

6. Camma C, Giunta M, Fiorica F, Pagliaro I, Craxi A, Cottone M. Preoperative radiotherapy for respectable rectal cancer: A meta-analysis. JAMA 2000; 284: 1008-15.

7. Glimelius B, Grönberg H, Järhult J, et al. A systematic overview of radiation therapy effects in rectal cancer. Acta Oncologica 2003; 42: 476-92.

8. Swedish Rectal Cancer Trial. Improved survival with preoperative radiotherapy in resectable rectal cancer. N Engl J Med 1997; 336: 980-7.

9. Swedish Rectal Cancer Trial. Long lasting benefits from radiotherapy on survival and local recurrence rate. J Clin Oncol 2005; 5644-50.

10. Lledó S. Cirugía Colorrectal. Guías Clínicas de la Asociación Española de Cirujanos. Madrid: Arán Ediciones; 2000.

11. Kim NK, Kim MJ, Yun SH, et al. Comparative study of transrectal ultrasonography, pelvic computerized tomography, and magnetic resonance imaging in preoperative staging of rectal cancer. Dis Colon Rectum 1999; 42 (6):770-5.

12. Vila JJ, Jiménez FJ, Irisarri A, et al. Estadificación del cáncer de recto mediante ultrasonografía endoscópica: correlación con la estadificación histológica. Rev Esp Enferm Dig 2007; 99: 132-7.

13. Bisset IP, Fernando CC, Hough DM, et al. Identification of the fascia propia by magnetic resonance imaging and its relevance to preoperative assessment of rectal cancer. Dis Colon Rectum 2001; 44 (2): 259-65.

14. Brown G, Radcliffe AG, Newcombe RG, et al. Preoperative of prognostic factors in rectal cancer using high resolution magnetic resonance imaging. Br J Surg 2003; 90 (3): 355-64.

15. MERCURY Study Group. Diagnostic accuracy of preoperative magnetic resonance imaging in predicting curative resection of rectal cancer: Prospective observational study. BMJ 2006; 333 (7572): 779.
16. Brown G, Davies S, Williams GT, et al. Effectiveness of preoperative staging in rectal cancer: Digital rectal examination, endoluminal ultrasound or magnetic resonance imaging? Br J Cancer 2004; 91: 23-9.

17. Palman L, Glimelius B. Pre o postoperative radiotherapy in rectal and rectosigmoid carcinoma. Report from a randomized multicenter trial. Ann Surg 1990; 211: 187-95.

18. Roh MS, Petrelli N, Wieand S, et al. Phase III randomized trial of postoperative trial of preoperative vs. postoperative multimodality therapy in patients with carcinoma of the rectum (NSABPR-03). Proc Am Soc Clin Oncol 2001; 20: A490.

19. Sauer R. Adyuvant versus neoadyuvant combined modality treatment for locally advanced rectal cancer: first results of the german rectal cancer study (CAO/ARO/AIO-94). Int J Radiat Oncol Biol Phys 2003; 57: S124-5

20. Kapiteijn E, Marijnen CA, et al. Preoperative radiotherapy combined with total mesorectal excision for resectable rectal cancer. N Engl J Med 2001; 345: 638-46.

21. Marijnen CA, Kapiteijn E, Van der Velde CJ, et al. Acute side effects and complications after short-term preoperative radiotherapy combined with total mesorectal excision in primary rectal cancer: Report of a multicenter randomized trial. J Clin Oncol 2002; 20: 817-25.

22. Frykholm GJ, Isacsson U, Nygard K, et al. Preoperative radiotherapy in rectal carcinoma: Aspects of acute adverse effects and radiation technique. Int J Radiat Oncol Biol Phys 1996; 35: 1039-48.

23. Peeters KCMJ, Van der Velde CHJ, Leer JWH, et al. Late side effects of short-course preoperative radiotherapy combined with total mesorectal excision for rectal cancer: Increased bowel dysfunction in irradiated patients -A Dutch Colorectal Cancer Group Study. J Clin Oncol 2005; 23 (25): 6199-206.

24. Birgisson H, Pahlman L, Gunnarson U, et al. Adverse effects of preoperative radiation therapy for rectal cancer: Long-term follow up of the Swedish Rectal Cancer Trial. J Clin Oncol 2005; 23 (34): 8697705 .

25. Heald RJ, Husband EM, Ryall RD. The mesorectum in rectal cancer surgery -the due to pelvic recurrence? Br J Surg 1982; 69: 613-6.

26. Marijen CA, Nagtegaal ID, Kranenbarg EK, et al. No downstaging after short-term preoperative radiotherapy in rectal cancer patients. J Clin Oncol 2001; 19: 1976-84.

27. Wheeler JMD, Warren BF, Mortensen NJMC, et al. Quantification of histologic regression of rectal cancer after irradiation. Dis Colon Rectum 2002; 45: 1051-6.

28. Minsky B, Cohen A, Enker WE, et al. Preoperative 5-FU, low dose leucovorin and radiation therapy for locally advanced and unresectable rectal cancer. Int J Radiat Oncol Biol Phys 1997; 37: 289-95.

29. Rich TA, Skibber JM, Ajani JA, et al. Preoperative infusional chemoradiation therapy for stage $\mathrm{T} 3$ rectal cancer. Int $\mathrm{J}$ Radiat Oncol Biol Phys 1995; 32: 1025-9.

30. Vecchio FM, Valentini V, Minsky B, et al. The relationship of pathologic tumor regression grade (TRG) and outcomes alter preoperative therapy in rectal cancer. Int J Radiat Oncol Biol Phys 2005; 62 (3): $752-60$.

31. Valentini V, Cocco C, Picciocchi A, et al. Does downstaging predict improved outcome alter preoperative chemoradiation for extraperitoneal locally advanced rectal cancer? A long term analysis of 165 patients. Int J Radiat Oncol Biol Phys 2002; 53 (3): 664-74.

32. Feliú J, Calvillo J, Escribano A, et al. Neoadyuvant therapy of rectal carcinoma with UFT-leucovorin plus radiotherapy. Ann Oncology 2002; 13: 730-6.

33. Janjan NA, Khoo VS, Abrucese J, et al. Tumor downstaging and sphincter preservation with preoperative chemoradiation in locally advanced rectal cancer: The MD Anderson Cancer Center experience. Int J Radiat Oncol Biol Phys 1999; 44 (5): 1027-38.

34. Minsky BD. Sphincter preservation for rectal cancer: Fact or fiction? J Clin Oncol 2002; 20: 1971.

35. Bujko K, Nowacki M, Nasierowska-Guttmejer A, et al. Sphincter preservation following preoperative radiotherapy for rectal cancer: Report of a randomised trial comparing short-term radiotherapy vs. conventionally fractioned radiochemotherapy. Radiother Oncol 2004; 72: $15-24$. 